\title{
The Clinical Area Safety Assessment (CASA), a peer review of safety across an acute trust
}

\author{
Susan Robinson ${ }^{1}$, J ames Ward ${ }^{2}$, Trevor Baglin ${ }^{1}$, Susan Broster ${ }^{1}$, Carol Heesom-Duff ${ }^{1}$, Glenn \\ Pascoe $^{1}$, J ag Ahluwalia ${ }^{1}$ \\ 1. Addenbrookes Hospital, Cambridge University Hospitals, Cambridge, United Kingdom. 2. Engineering Design Centre, \\ Department of Engineering, University of Cambridge, Cambridge, United Kingdom \\ Correspondence: Susan Robinson. Address: The Cambridge Patient Safety Unit, Cambridge University Hospitals \\ Foundation Trust, Hills Road, Cambridge, CB2 0QQ, United Kingdom. E-mail: Susan.robinson@addenbrookes.nhs.uk
}

Received: October 15, 2012

Accepted: December 4, 2012

Online Published: December 24, 2012

DOI : $10.5430 /$ jha.v2n2p27

URL: http://dx.doi.org/10.5430/jha.v2n2p27

\section{Abstract}

Background: Most hospitals have developed processes to manage risk in a reactive manner. Few, however, have instituted proactive systems for the identification of latent risk that has the potential to cause harm.

Objective: To develop a process for the identification of potential risks to safety across all clinical areas of a hospital.

Methods: Every clinical department in Cambridge University Hospitals Foundation Trust (CUHFT) underwent peer assessment to confirm that the Trust's processes for safety were in place and identify possible threats to the safety of patients. This assessment consisted of a number of elements that included review of routinely collected data, observed clinical care and a safety questionnaire. The methodology used to apply this process is described.

Results: The outcomes of 33 clinical area safety assessments (CASA) are reported. No department was awarded unconditional accreditation nor have any had their service suspended. A number of recurrent issues emerged, the most common being that $31 \%$ of departments failed to fully comply with Trust requirements for governance and $12 \%$ needed to improve compliance with patient safety standards. Concerns related to documentation were identified in $11 \%$ of assessments. To date the programme has cost approximately £111,000 with each review requiring approximately 130 hrs to complete.

Conclusions: The CASA programme has offered an opportunity to improve standardisation in governance and optimise safety processes across our hospital. It has facilitated the dissemination of good practice amongst teams to resolve common problems. Suggestions as to how we plan to further refine this peer review process are offered.

\section{Key words}

Patient safety, Peer review, Safety assessment

\section{Introduction}

To Err is Human, published in 1999, was uncompromising in its assessment of the level of safety within healthcare ${ }^{[1]}$. Subsequently, An Organisation with a Memory ${ }^{[2]}$ and Building a Safer NHS for Patients ${ }^{[3]}$ both highlighted the need to learn as a result of things going wrong. It is known that the most effective ways to reduce error and harm is to target 
underlying system failures ${ }^{[1,2,4-7]}$. However whilst most hospitals have developed processes to manage risk in a reactive manner, few have attempted to develop proactive systems for the identification of risk that has the potential to result in harm within their organisation.

Like many hospitals, CUHFT had introduced safety walkabouts in an attempt to be more proactive. Walkabouts have been shown to increase senior executive's understanding of safety concerns and the problem from a systems perspective ${ }^{[8]}$. More recently, they have been associated with an improvement in the safety climate scores of some health organisations ${ }^{[9]}$.

It was accepted our hospital was effective at investigating safety issues once they had occurred but concerns were raised that, despite walkabouts, it was difficult to be confident that all areas of the Trust were safe. Specifically there was a requirement to establish a process of assurance that all areas were compliant with Trust safety standards and governance processes and were monitoring their outcomes in terms of safety and quality at ward and department level.

The majority of NHS policy regarding safety has focused at the organisational level and that of the individual provider whereas systems theory focuses on structures and the reliability of the organisation. Nelson et al described the elements of a microsystem as a core team of healthcare professionals working together with the shared purpose of delivering care to a defined group of patients, supported by information and an appropriate work environment ${ }^{[10]}$. These elements are well aligned to the characteristics of a clinical department. These authors described how a focus on microsystems provides an opportunity to improve standardisation and improvements in performance and teamwork across large organisations.

The final recommendation of the four made by the IOM report in $1999^{[1]}$ was to create safety systems inside healthcare organisations through the implementation of safe practices at the delivery level. More recently Ikkersheim and Berg identified four interconnected elements that were crucial for hospital reliability. These were: 1 . Process optimisation and standardization; 2. Outcome measurement and monitoring; 3. Responsibilities and accountability of medical professionals; and 4. Organizational culture ${ }^{[11]}$. It is only by recognising where the deficiencies are within an organisation can one improve reliability by focusing efforts on those areas where improvement is required.

It was proposed a more proactive system for the identification of potential safety problems within clinical areas be developed so that these could be addressed prior to a significant incident occurring. In addition emerging themes could be collated and used to inform future programmes of work, strategy or priorities for investment. This programme is known as The Clinical Area Safety Assessment (CASA) programme. It is a proactive peer assessment of patient safety across the hospital and was designed to confirm the Trust's systems and processes regarding safety were embedded within each department so reducing risks to the safety of patients. The purpose of this paper is to describe the methodology used, outline the costs associated with the assessments and share our experience of running the CASA programme.

\section{Methods}

\subsection{Background}

Cambridge University Hospitals (CUHFT) has approximately 1,000 beds in total, employs over 7,000 staff and offers the full range of local and tertiary services other than cardio-thoracic surgery. Its maternity hospital delivers 5,800 babies a year. Clinical care is delivered by 7 divisions and 45 clinical directorates, all supported by a single corporate division.

The structure for safety within the Trust includes The Patient Safety Unit (PSU) which combines the roles of the patient safety, clinical risk and audit. Patient Safety Managers, each with a small support team, work with the Divisions and Clinical Departments in all areas related to Patient Safety. A dedicated information analyst supports these staff. The Patient Safety Executive is the committee with operational responsibility for ensuring and improving patient safety. The 
Trust has clear expectations in regard to how governance should be managed and has a mechanism for communicating specific safety standards across the Trust, called patient safety directives.

\subsection{The CASA process}

The review process described in this paper started in December 2009 and finished in January 2012. The assessment consisted of a number of different elements. Each was identified as being key to facilitating an understanding of the area under review and relevant to the identification of risk across the two hospitals. A number of elements included data already collected by the Trust as part of its formal governance and risk systems. Staff from all levels of the organisation who attended a multidisciplinary workshop informed the choice of criteria to be collected and reviewed as part of this process. These were then refined by the (then) clinical governance team and were first piloted with two clinical departments (Medicine for the Elderly and Gynaecology) to confirm their relevance, usability and acceptability. This pilot highlighted the need to collect the data centrally rather than rely on the clinical area under review to complete this task and emphasised the importance of ensuring any additional work presented to the clinical teams by this process was kept to a minimum.

Table 1. CASA Process

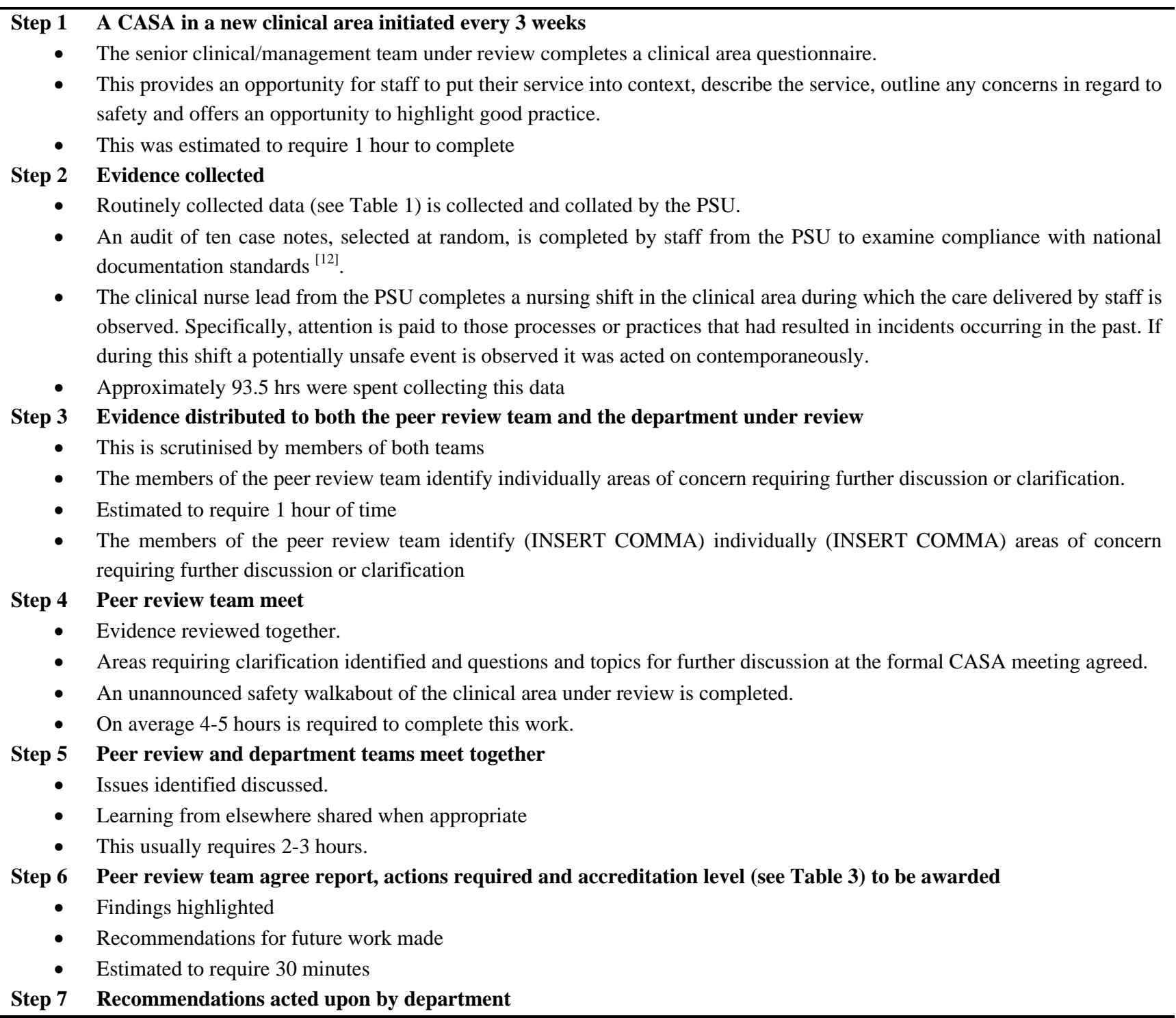


The different methods used to assess safety and the sequence of events in the CASA process are summarised in Table 2. An assessment (peer review) team consisted of a consultant, a senior nurse and a senior manager, all from a different clinical area to that under review. The Head of Patient Safety, who ensured consistency of assessment, accompanied these staff.

Table 2. Routine data reviewed by the CASA Process

\begin{tabular}{|c|c|}
\hline Routine collected data (Triggers) & Detail \\
\hline NPSA Never Events & Occurrence of any \\
\hline \multirow{5}{*}{$\begin{array}{l}\text { Addenbrookes Never Event (a near miss which if } \\
\text { allowed to progress could result in a never or serious } \\
\text { event) }\end{array}$} & Incorrect patient crossing the threshold of theatre (reception) \\
\hline & Elective surgery without appropriate marking \\
\hline & Issue of blood product to wrong patient \\
\hline & Pressure ulcers grade 2 or above (acquired as in patient) \\
\hline & Mislabelled patient \\
\hline Mortality & Mortality rate for clinical specialty \\
\hline Medication related incidents & Number and severity \\
\hline Patient falls & Number and severity \\
\hline \multirow{7}{*}{ Infection control } & Hand hygiene compliance rates \\
\hline & Intravascular cannula care \\
\hline & Urinary catheter care \\
\hline & MRSA decolonisation \\
\hline & Acquired Clostridium Difficile \\
\hline & MRSA Bacteraemia (hospital acquired) \\
\hline & Presence of divisional infection control meetings \\
\hline \multirow{8}{*}{ Risk Management } & Number and severity of incidents \\
\hline & Unplanned admission to critical care area \\
\hline & Number of crash calls \\
\hline & Readmission within 30 days to same specialty \\
\hline & Development of deep vein thrombosis (DVT) or pulmonary embolus (PE) \\
\hline & as a result of admission \\
\hline & Compliance with venothromboembolism (VTE) assessment \\
\hline & Risk assessments all in date and actions being addressed where appropriate \\
\hline \multirow[t]{2}{*}{ Governance meeting } & Quarterly meetings held following Trust template \\
\hline & Expected nursing hours per patient day (NHPPD) fulfilled \\
\hline \multirow{4}{*}{ Staffing } & No of staffing related incidents \\
\hline & All staff up to date with competency and mandatory training \\
\hline & All clinicians have professional registration up to date \\
\hline & Evidence of investigation and action plans of complaints selected \\
\hline Communications & Patient and staff feedback results \\
\hline Local triggers & Local to specialty e.g. time to CT for stroke \\
\hline
\end{tabular}

It was originally anticipated that each department would undergo at least one assessment over a 2 year period. However the safety of all clinical departments is continuously monitored by regular review of the trigger data detailed in Table 1 and if necessary an earlier or repeat review would be organised.

The CASA reports were to be used locally to implement change and so improve safety. Whilst each clinical department was responsible for the implementation of any actions required, progress was monitored via the Patient Safety Executive, through the Patient Safety Unit. Whilst the reports were to be discussed at local clinical governance meetings the exact process for dissemination within individual teams varied between different clinical teams. All reports are available on the 
CUHFT’s central server. Department CASA summaries also form part of the Quarterly Patient Safety Report presented by the Medical Director to the Quality Committee and Trust Board.

Table 3. Accreditation Level of Safety following the CASA

\begin{tabular}{|c|c|c|}
\hline Level & Definition & Example \\
\hline Unconditional accreditation & No concerns regarding safety & $\begin{array}{l}\text { Not applicable } \\
\text { Addition of clinical governance minutes to Trust server }\end{array}$ \\
\hline $\begin{array}{l}\text { Conditional accreditation } \\
\text { level } 1\end{array}$ & $\begin{array}{l}\text { Simple changes required to improve } \\
\text { safety }\end{array}$ & $\begin{array}{l}\text { Mandatory training not up to date } \\
\text { Ward fridges require locking } \\
\text { Cleanliness of wards } \\
\text { Management of team behaviours required }\end{array}$ \\
\hline $\begin{array}{l}\text { Conditional accreditation } \\
\text { level } 2\end{array}$ & $\begin{array}{l}\text { More complex interventions } \\
\text { required to improve safety }\end{array}$ & $\begin{array}{l}\text { Concerns regarding out of hours care } \\
\text { Establishment of formal mortality and morbidity (M\&M) } \\
\text { meetings required }\end{array}$ \\
\hline Suspension & $\begin{array}{l}\text { This could mean suspension of an } \\
\text { activity within a service or the } \\
\text { entire service }\end{array}$ & $\begin{array}{l}\text { Running a service on dangerously low levels of staff with } \\
\text { inadequate skill mix. } \\
\text { Breakdown in a process likely to result in significant harm. }\end{array}$ \\
\hline
\end{tabular}

\section{Results}

\subsection{Level of safety}

To date, of 33 assessments, 15 areas have been assessed at level 1 and 18 at level 2. No department has received unconditional accreditation nor have there been any suspensions of activity or service.

A number of themes have emerged (see Table 4). Over 30\% of the concerns identified relate to a failure of the clinical and managerial teams to comply with Trust requirements for Governance; in particular the formal review of incidents, infection control and quality metrics. This was identified by review of the minutes of the department's clinical governance, safety, infection control and M\&M meetings. In addition failure to ensure widespread multidisciplinary attendance at such meetings was evident. The quality of case review within M\&M meetings was sub-optimal as was the use of action logs to ensure completion of actions identified. Poor compliance with specific Trust patient safety standards, issued as Patient Safety Directives or Alerts, featured in $12 \%$ of the concerns raised whilst poor documentation was similarly represented. Clinical teams in addition to the peer review team highlighted inadequate staffing levels, particularly out of hours. Inadequate completion of mandatory training by staff was also identified within some departments. Concerns regarding poor teamwork were encountered or identified less frequently.

\subsection{Costs}

The CASA programme is not without cost. Each individual CASA has cost approximately $£ 3,500$ and to date the whole programme has cost the Trust an estimated $£ 111,000$. The breakdown of costs is shown within Table 5. These figures are based on the hours spent by key participants in the CASA process with salary costs for a consultant based on the mid level of a basic salary. On average each CASA required 130 hrs. Whilst the number of hours required may appear significant, when compared to the time reported as necessary to complete a root cause analysis (RCA) 130 hrs may become more acceptable. Mills et al reported that a RCA of 143 single incidents related to adverse drug events took a median of 35 person hours (range 6-1,590 hours) ${ }^{[13]}$. Mills also reviewed 176 aggregate RCA on patient falls and identified each one required 47.80 hours on average (SD $32.40 \mathrm{hrs)}{ }^{[14]}$. Given the CASA process was broader than a typical RCA it is understandable it takes longer. 
The costs do not reflect any savings incurred by the prevention of harm or efficiencies made by improvements in teamwork or process as a result of the CASA review. Whilst it is difficult to measure whether the benefits of this specific programme are worth the financial cost, there is a sense the CASAs have contributed to the heightened awareness of safety across the trust and successful achievement of Clinical Negligence Scheme for Trusts (CNST) Level 3 in the past few months (the CNST manages all clinical negligence claims against member NHS bodies where the incident in question took place on or after 1 April 1995).

Table 4. Areas Identified by the CASA Process as Requiring Improvement

\begin{tabular}{|c|c|}
\hline Areas Requiring Improvement & Proportion of all Areas (Rank*) \\
\hline $\begin{array}{l}\text { Organisation and process of governance - follows Trust policy in terms of safety and } \\
\text { governance }\end{array}$ & $31 \%(1)$ \\
\hline e.g. Improve use of action logs to ensure all actions are completed & \\
\hline $\begin{array}{l}\text { Trust patient safety standards - compliance with all patient safety directives and alerts } \\
\text { e.g. Improve compliance with requirement all admissions seen by consultant within } \\
24 \text { hrs }\end{array}$ & $12 \%(2)$ \\
\hline $\begin{array}{l}\text { Documentation - level of compliance with national standards of documentation } \\
\text { e.g. Legibility of medical entries requires improvement }\end{array}$ & $11 \%(3)$ \\
\hline $\begin{array}{l}\text { Staffing levels - according to locally agreed nursing hours per patient day (NHPPD) } \\
\text { e.g. Staffing for acuity of patients’ needs to be reviewed }\end{array}$ & $10 \%(4)$ \\
\hline $\begin{array}{l}\text { Staff training - evidence of competence assessment and compliance with mandatory } \\
\text { training }\end{array}$ & $8 \%(5)$ \\
\hline e.g. Assurance required that all nursing staff trained in resuscitation & \\
\hline $\begin{array}{l}\text { Teams - evidence of effective team working both within clinical teams and different } \\
\text { teams }\end{array}$ & $5 \%(6)$ \\
\hline e.g. Information sharing needs to be improved & \\
\hline $\begin{array}{l}\text { Administrative process - efficiency of operational processes key to delivering timely, } \\
\text { safe care }\end{array}$ & $4 \%(7)$ \\
\hline e.g. Delay to follow up of patients to be addressed & \\
\hline $\begin{array}{l}\text { Facilities - availability of appropriate facilities/estate to facilitate safe care } \\
\text { e.g. The environment of clinic to support efficiency and safety should be reviewed }\end{array}$ & $3 \%(8)$ \\
\hline $\begin{array}{l}\text { Housekeeping - adequacy of cleaning and restocking to ensure timely, safe care } \\
\text { e.g. Address ward cleanliness }\end{array}$ & $3 \%(8)$ \\
\hline $\begin{array}{l}\text { Handover - evidence of use of formal systems for handover of patients } \\
\text { e.g. Measures for patient handover need to be improved }\end{array}$ & $2.5 \%(9)$ \\
\hline $\begin{array}{l}\text { Progress on safety concerns - evidence of processes and procedures to ensure all } \\
\text { safety concerns are addressed } \\
\text { e.g. Address lack of action on management of abnormal blood results }\end{array}$ & $2.5 \%(9)$ \\
\hline $\begin{array}{l}\text { Capacity - availability of sufficient capacity for numbers and acuity of patients } \\
\text { e.g. Review safety of using ward as contingency area }\end{array}$ & $2 \%(10)$ \\
\hline $\begin{array}{l}\text { Clinical care - use of effective care pathways and compliance with national standards } \\
\text { of care }\end{array}$ & $2 \%(10)$ \\
\hline e.g. Improve compliance with Ventilator Associated Pneumonia Bundle & \\
\hline $\begin{array}{l}\text { Patients - no of complaints and level of patient satisfaction } \\
\text { e.g. Concerns raised regarding patient experience survey results }\end{array}$ & $1.5 \%(11)$ \\
\hline $\begin{array}{l}\text { Service improvement - evidence of engagement to improve the quality of care } \\
\text { e.g. Look to improving pathways of care for abdominal pain from ED to specialty } \\
\text { Culture - evidence of awareness and application of safety principles }\end{array}$ & $1 \%(12)$ \\
\hline $\begin{array}{l}\text { e.g. The department perceives they are a low risk specialty and therefore have not } \\
\text { identified the need for assurance in regard to patient safety }\end{array}$ & $1 \%(12)$ \\
\hline $\begin{array}{l}\text { Sickness - level of sickness rates and process to manage } \\
\text { e.g. Improve management of sickness }\end{array}$ & $0.5 \%(13)$ \\
\hline
\end{tabular}


Table 5. Costs of Peer Assessment Process

\begin{tabular}{|c|c|c|c|c|c|}
\hline \multicolumn{2}{|c|}{ Patient Safety Team } & \multicolumn{2}{|c|}{ Peer Review Team } & \multicolumn{2}{|c|}{ Clinical Team } \\
\hline Band*3 & $2 \mathrm{hrs}$ & Consultant & $7 \mathrm{hrs}$ & Consultant & 5hrs \\
\hline Band 5 & $27 \mathrm{hrs}$ & Band 8A & $7 \mathrm{hrs}$ & SCN & $5 \mathrm{hrs}$ \\
\hline Band 6 & 16 hrs & Band 8B & $7 \mathrm{hrs}$ & Dept Manager & 5 hrs \\
\hline Band 7 & 34 hrs & & & & \\
\hline Band $8 \mathrm{~A}$ & $7.5 \mathrm{hrs}$ & & & & \\
\hline Band 8C & 7 hrs & & & & \\
\hline Sub-total & 93.5hrs & & $21 \mathrm{hrs}$ & & $15 \mathrm{hrs}$ \\
\hline \multicolumn{2}{|l|}{ Total } & \multicolumn{4}{|l|}{ 129. $5 \mathrm{hrs}$} \\
\hline \multicolumn{2}{|c|}{ Cost per CASA } & \\
\hline \multicolumn{2}{|c|}{ Cost to date } & \multicolumn{4}{|l|}{$\begin{array}{l}£ 3,359 \\
£ 110,858\end{array}$} \\
\hline
\end{tabular}

${ }^{*} \mathrm{~A}$ band is equivalent to the grade of staff. It is the term used in the UK and reflects the pay scale.

\subsection{Qualitative data on learning and behaviour change}

The opportunity for external scrutiny, in particular the chance to raise and discuss local concerns regarding safety, has been welcomed by most participants. Those participating in the peer assessment team report they personally gained from the process and report lessons learnt by reviewing another area are taken back to their own clinical team.

The obvious tensions that exist between and within a few teams have taken some of the peer review members by surprise although the CASA has provided a safe environment in which to explore legitimate concerns and on occasion challenge what occurs locally. In some cases there was robust challenge amongst peers regarding the commonly held view by some that clinical staff can do little to improve safety.

Incidents rarely involve a single speciality so working together to investigate and improve processes and systems is key. The PSU report an increase in the frequency and the quality of communication between disciplines in identifying and reaching agreement as to the changes required to improve safety.

Areas of good practice were identified during the process and have been highlighted across the organisation to aid the resolution of often, common problems; for example, the provision of local induction packs to junior medical staff before commencing work.

Routine review of our safety metrics shows an increase in reported incidents from 2009/10 to 2011/12 of 6.6\%. This increase is on a background of reduced capacity and an increase in the acuity of patients admitted to CUHFT although there has been no significant increase in the numbers of patients being admitted. However the proportion of those incidents resulting in harm has gone up by $38.5 \%$ largely as a result of a change in the national reporting requirements for pressure ulcers.

The absolute requirement to address all actions identified as a result of an incident or review of care is now more widely accepted by staff; although oversight of this by the Patient Safety Executive remains.

\subsection{Lessons for others}

The peer review process should be seen as one piece of the puzzle in understanding and improving safety across an organisation. It needs to sit within an organisational structure that is capable of providing safety related data as well as 
ensuring all actions identified as being necessary are completed. The organisation needs to be transparent as to its standards in terms of safety and governance and these need to be well established prior to any assessment.

Staff require time to participate in this process and so it must be resourced appropriately in terms of both time and staff. Clinical and managerial leaders need to ensure the work done as part of this process is recognised as important.

This process provided an overview and an opportunity to review local safety data but on reflection did not allow a detailed exploration of process or culture. Some months after completing the CASA process for one department 3 never events occurred in this area over a very short space of time. In this regard the CASA process may have resulted in a false negative result because review of the department’s CASA revealed no evidence that something was amiss.

Consideration should be given to the coupling of the peer review team with the clinical team being assessed. We chose to do this in such a way as to promote team working between related areas e.g. Paediatric Intensive Care and the Emergency Department. It is important to note that teams should not assess each other; the pairings for all CASAs must be different although a consistent approach is vital to ensure fairness and an equitable assessment, without this staff will not engage with the review.

\subsection{Limitations of the data}

Our aim was to reduce the potential for harm to occur within our hospitals. It is difficult to specifically measure the effect on harm that has not yet occurred. Formal evaluation is required to quantify the changes in learning and behaviour that have been reported by our staff.

The CASA programme is just one part of a multifaceted Trust-wide approach to improving safety so it is difficult to ascribe any improvement in safety as being solely due to the CASA process. It is possible that some aspects of the review process, particularly the walkabouts and observation of practice, were subject to a Hawthorne effect given those involved knew their department was being examined. Clinical staff are used to the PSU staff being present in all areas of the hospital so we hoped this effect would be minimal. Any Hawthorne effect may have been offset by the fact other methods of collecting data were used.

\subsection{Future developments}

Future changes to the CASA process will aim to foster additional developments known to enhance reliability and quality within clinical departments. One could also argue for the necessity of including non-clinical departments to ensure alignment of all microsystems in delivering safer care.

The measures used in this review are not new and in many instances look for the presence of something tangible, a process or compliance with a procedure rather than the quality of the action. For example, the documentation audit considered legibility but not the effectiveness of entries e.g. were all actions documented as necessary within the notes actually completed. In the next reiteration of the peer review process we need to identify a system for assessing quality, for example, by assessing the quality of care or evidence of harm in the case note review rather than the adherence to national documentation standards.

Ensuring all staff participate in the assessment of our safety culture has proved challenging to date so using the CASA process to engage with individual staff to collect high quality information regarding culture, behaviours and teamwork via formal qualitative one to one interviews is likely to be a feature. Safety is known to be adversely affected by conflict within teams, individual poor behaviour and a failure to adhere to organisational values. Accurate information regarding the presence of such negative attributes needs to be acquired. Most individuals are well versed in the use of individual $360^{\circ}$ appraisal; perhaps it is time to consider $360^{\circ}$ appraisals of entire teams or departments. 
A number of recurrent problems were identified as a result of undertaking the CASAs. In future consideration must be given to effectively managing these risks by the use of techniques such as Prospective Hazard Analysis as discussed by Ward et al ${ }^{[15]}$. In future CASAs it may be appropriate to focus on the key risk identified by the clinical team under review e.g. a never event and work with the team using a technique such as PHA to reduce the risk of occurrence. This work would be likely to have system wide applicability.

To date we have been unable to describe an appropriate methodology to formally evaluate the effectiveness of CASA process over time. We need to address the development of these metrics for the second round although the challenge of doing so is well recognized ${ }^{[16]}$. Furthermore it would be useful to establish a formal mechanism for evaluating satisfaction with the peer review process.

\section{Conclusions}

Our aim in developing this internal peer review of safety was to ensure all areas complied with the Trust's systems and processes regarding safety and facilitate a change in safety behaviour and practice through external scrutiny, in addition to identifying potential hazards prior to them resulting in harm. Prior to the CASA process it was assumed that all areas were compliant with Trust standards and processes and were monitoring their outcomes in terms of safety and quality. The fact this was not the case was a significant learning point for our organisation and signals we have much work to do in ensuring compliance with future safety standards. The CASA review has enabled the patient safety unit to acquire a view of the degree of adherence to trust safety standards but perhaps more importantly it has ensured all clinical teams are aware of the expectations in this regard. This first phase of review has ensured every department meets a minimal standard in terms of implementing safe practices.

Mohr ${ }^{[17]}$ described eight characteristics of effective microsystems; a number of these were identified as deficient in some areas of the Trust whilst well advanced in other clinical departments. We believe our process has increased awareness amongst staff as to how a well-functioning department can contribute to the safety of care within an organisation. We hope that future staff surveys will include appropriate questions to measure this effect.

Pronovost recently discussed how the widespread application of a process similar to the peer-to-peer assessment programme currently used in the nuclear industry should be considered in order to identify safety hazards and share best practice in terms of improving safety and operational performance between different hospitals ${ }^{[18]}$. We are unaware of other hospitals using team-to-team peer review in an effort to improve safety although organisational peer review has been used to improve the care of patients with chronic obstructive lung disease ${ }^{[19]}$.

We would suggest that the advantages of the CASA process we have developed is that it is a proactive, multi-faceted process that provides a mechanism for systematically evaluating the safety of clinical departments by peers from a variety of disciplines.

One of the greatest values of the CASA process has been the discussion with staff, which has an important benefit of getting staff to think about the safety in their area of work.

The CASA programme has enabled the Trust to understand the level of safety within individual areas of the hospital and has provided an opportunity to identify those areas that have not instituted the Trust's minimal standards or expected processes for monitoring, reviewing and learning from harm events. Perhaps most importantly the CASA has promoted an open discussion about what we haven't got right and where we need to improve. 


\section{Acknowledgements}

The authors acknowledge the work done by staff within the Patient Safety Unit to develop and complete the CASA programme. We also thank all our clinical and managerial colleagues who volunteered to participate in the peer review teams. We thank Professor Martin Bobrow, Non-Executive Director and Chair of the Governance Committee for his thoughtful review of this paper.

\section{Competing interests}

All authors declare that there was no support from any organisation, other than CUHFT, for the submitted work and no relationships with any organisations that could have influenced the submitted work.

\section{Funding}

The Cambridge Safety Unit is a partnership between Cambridge University Hospitals Trust and the Engineering Design Centre, University of Cambridge. This work was funded by CUHFT.

\section{Contributions}

TB, CHD, JA and GP contributed to the concept and design of the original peer review programme. TB, CHD, SB and SR all participated in the peer review process. JW contributed to the critical review of the programme whilst SR drafted the initial draft of the manuscript and completed the data analysis and interpretation of findings. All authors contributed to the refinement and further revision of the manuscript. All approved the final version.

\section{References}

[1] Kohn LT, Corrigan JM, Donaldson, MS, Eds. To Err is Human: Building a Safer Health System. Washington, D.C. Institute of Medicine Report. National Academy Press. 1999.

[2] Department of Health. An Organisation with a Memory. London. HSMO. 2000.

[3] Department of Health. Building a safer NHS for patients - implementing an organisation with a memory. London. HSMO. 2001.

[4] Van der Schaaf TW. Medical applications of industrial safety science. Qual Saf Health Care. 2002; 11: 205-206. PMid:12486976 http://dx.doi.org/10.1136/qhc.11.3.205

[5] McGinnis L. Creating high reliability: a new approach for patient safety. AORN Journal. 2011; 94: 219-222. PMid:21884839 http://dx.doi.org/10.1016/j.aorn.2011.05.016

[6] Leischow SJ, Best A, Trochim WM et al. Systems thinking to improve the public's health. Am J Prev Med. 2008; 35(2 Suppl): S196-203. PMid:18619400 http://dx.doi.org/10.1016/j.amepre.2008.05.014

[7] Emslie S. Building a safer NHS for patients. In Improving Patient Safety: Insights from American, Australian and British Healthcare. ECRI and Department of Health. 2002.

[8] Frankel A, Graydon-Baker E, Neppl C et al. Patient Safety Leadership WalkRounds. Joint Commission Journal on Quality and Safety. 2003; 29: 16-26. PMid:12528570

[9] Frankel A, Pratt Grillo S, Pittman M et al. Revealing and resolving patient safety defects: the impact of leadership WalkRounds on frontline caregiver assessments of patient safety. Health Ser Res. 2008; 43: 2050-2066. PMid:18671751 http://dx.doi.org/10.1111/j.1475-6773.2008.00878.x

[10] Nelson EC, Batalden PB, Mohr JJ et al. Building a quality future. Frontiers Health Serv Manage. 1998; 15: 3-32. PMid:10182606

[11] Ikkersheim DE, Berg M. How reliable is your hospital? A qualitative framework for analysing reliability levels. BMJ Qual Saf. 2011; 20: 785-790. PMid:21441603 http://dx.doi.org/10.1136/bmjqs.2010.045187

[12] Academy of Medical Royal Colleges. A Clinician's Guide to Record Standards - Part 2: Standards for the structure and content of medical records and communications when patients are admitted to hospital. London. Royal College of Physicians. 2008.

[13] Mills PD, Neily J, Kinney LM et al. Effective interventions and implementation strategies to reduce adverse drug events in the Veterans Affairs (VA) System. Qual Saf Health Care. 2008; 17: 37-46. PMid:18245218 http://dx.doi.org/10.1136/qshc.2006.021816

[14] Mills PD, Neily J, Luan D et al. Using aggregate root cause analysis to reduce falls and related injuries. Jt Comm J Qual Patient Safety. 2005; 31: 21-31. 
[15] Ward J, Clarkson J, Buckle P, et al. Prospective Hazard Analysis:Tailoring Prospective Methods To A Healthcare Context [Internet]. Research. Project PS/035 Report to the Patient Safety Research Programme of the Department of Health. 2010. Available from:

http://www.haps2.bham.ac.uk/publichealth/psrp/documents/PS035_-Revised_PHA_Final_Report_v1.1_with_Toolkit_July_201 $0 \_. p d f$

[16] Inside your Hospital. Dr Foster Hospital Guide 2001-2011. Dr Foster Intelligence. November 2011

[17] Mohr JJ, Batalden PB. Improving safety on the frontlines: the role of clinical Microsystems. Qual Saf Health Care. 2002; 11: 45-50. http://dx.doi.org/10.1136/qhc.11.1.45

[18] Pronovost PJ, Hudson DW. Improving health care quality through organisational peer-to-peer assessment: lessons from the nuclear power industry. BMJ Qual Saf Published Online First: 5 May 2012.

[19] Roberts CM, Buckingham RJ, Stone RA et al. The UK National Chronic Obstructive Pulmonary Disease Resources and Outcomes Project - a feasibility study of large scale clinical service peer review. J Eval Clin Pract. 2010; 16: 927-32. PMid:20557406 http://dx.doi.org/10.1111/j.1365-2753.2009.01224.x 